\title{
STRUCTURE OF AQUEOUS SOLUTIONS OF TETRAMETHYLAMMONIUM CHLORIDE INVESTIGATED BY POSITRON ANNIHILATION AND ULTRASONIC METHODS
}

\author{
G. JÁKLI ${ }^{a}$, K. JERIE ${ }^{b *}$, A. BARANOWSKI ${ }^{b}$ AND J. GLIŃSKI ${ }^{c}$ \\ ${ }^{a}$ KFKI A tomic Energy Research Institute, Budapest, Hungary \\ ${ }^{b}$ Institute of Experimental Physics, Wrocław University \\ Cybulskiego 36, 50-205 Wrocław, Poland \\ ${ }^{c}$ Faculty of Chemistry, Wroctaw University \\ F. Joliot-Curie 14, 50-383 Wrocław, Poland
}

(Received October 9, 1997; revised version December 12, 1997)

\begin{abstract}
The structure of aqueous solutions of tetramethylammonium chloride was investigated using sound velocity and positron annihilation methods. The results are different than those obtained earlier for systems where hydrophobic hydration occurs, although some evidences for formation of clathrate-like hydrates in liquid phase were observed. The results are interpreted, among others, in terms of competition of different hydrates of tetraalkylammonium cations, hydration of chloride anions, and formation of ionic pairs.
\end{abstract}

PACS numbers: $61.25 . \mathrm{Em}, 78.70 . \mathrm{Bj}$

\section{Introduction}

The structure of water is known to be strongly influenced by addition of solutes, both hydrophobic and hydrophilic. It was shown in our previous papers (cited below) that the long-lived component of the positron annihilation spectrum exhibits characteristic features close to the composition of mixture corresponding to that of hydrates. In particular, the long-lived component's lifetime passes through a plateau, and its intensity - through a maximum at concentration corresponding roughly to the stoichiometry of the clathrate-like hydrate formed in liquid solution. Moreover, the same stoichiometry could be extracted from the interception point of compressibility isotherms. The above was well confirmed in series of aqueous solutions of normal and isomeric short-chained alcohols [1], as well as in the system water + tetramethylurea [2].

Organic electrolytes, in particular tetraalkylammonium cations, $\left(\mathrm{C}_{n} \mathrm{H}_{2 n+1}\right) \mathrm{N}^{+}$, are of special interest because of their size and charge. In solid state they are supposed to form crystalline-like hydrates similar structurally to

*Corresponding author. 
the clathrates known for many nonelectrolytes, but stoichiometries of these hydrates are different from the known ones [3] (for details on clathrate hydrate stoichiometries see, for example, the review of Byk et al. [4]). At present, however, it is not yet clear what kind of water structure is formed around the alkyl groups of the large cations: ice-I-like, gas-hydrate-like, a structure involving some non-tetrahedral H-bonds, or possibly mixtures of all these [5]. On the other hand, the structure of aqueous solutions of tetramethylammonium salts is exceptional among the tetraalkylammonium salts, most probably because of the relatively small size of the cation (if compared to other salts of this type).

The effect of tetraalkylammonium salts on structure of water was, among others, the subject of the review of Huot and Jolicoeur [6], where the structure-breaking character of these solutes was proved on the basis of different experimental methods (spectroscopic, conductometric etc.) and theoretical considerations.

In their classical paper, Frank and Evans [7] concluded that the large negative entropies of dissolution of apolar solutes (in partic!llar gases) in water could be explained by the formation of "microscopic icebergs" around the solute molecules. Also the solvation energies of monovalent ions could be explained by a breaking-down of hydrogen-bonded structure of water. This concept was extended later by Frank and Wen [8] to explain the singular heat capacity behaviour of tetraalkylammonium salts in water (short-lived aggregates of water molecules are in equilibrium, and exchange rapidly with monomers). The above was later supported by the occurrence of clathrate hydrates for many apolar and polar solutes and organic electrolytes [9].

In this paper the system water + tetramethylammonium chloride, $\left(\mathrm{CH}_{3}\right)_{4} \mathrm{~N} \cdot \mathrm{Cl}$, was investigated using three independent methods: sound velocity, volumetry, and positron annihilation. The goal was to find out if the tetramethylammonium cations, $\left(\mathrm{CH}_{3}\right)_{4} \mathrm{~N}^{+}$, form inclusion-type hydrates in liquid aqueous mixtures.

\section{Experimental}

Chemicals. Tetramethylammonium chloride (Merck, pure for synthesis) was crystallized from methanol (annihilation and acoustic measurements) or from water + methanol + ether mixtures (density) and dried under vacuum. Water was doubly distilled. Solutions were prepared by weighing.

Positron annihilation measurements. The annihilation measurements were carried out at room temperature (approximately $20^{\circ} \mathrm{C}$ ) with a standard measuring device based on the "fast-slow" coincidence technique as described elsewhere [10]. Solutions were refluxed with nitrogen before the measurements to remove oxygen dissolved in the samples. The obtained spectra were resolved using POSITRONFIT program.

Sound velocity measurements. The ultrasonic velocity was measured at 15 , $20,25,30$, and $35^{\circ} \mathrm{C}\left( \pm 0.01^{\circ} \mathrm{C}\right)$ with an accuracy of $\pm 0.1 \mathrm{~m} / \mathrm{s}$ using a "sing-around" equipment (apparatus Ecolab MPFU, Poland). The details of the apparatus and measurement techniques applied in this study are given in [11].

Density measurements. Densities were measured at the same temperatures as sound velocity using Anton-Paar vibrating tube densitometer type DMA 60/602 H 
(each test was repeated twice). The calibration was determined daily using the known densities of air and $\mathrm{H}_{2} \mathrm{O}$. The reproducibilities of the densities were 1-2 ppm.

\section{Results}

\subsection{Density}

The plots of density on molar concentration of tetramethylammonium chloride in water were fitted using the following equation:

$$
\frac{d-d_{0}}{m}=A_{0}+A_{1} m^{\frac{1}{2}}+A_{2} m+A_{3} m^{\frac{3}{2}}
$$

(where $d$ and $d_{0}$ - the densities of a solution and pure solvent, respectively, $m$ - molality of the solute [moles solute $/ \mathrm{kg}$ of solvent]). The parameters are collected in Table I.

TABLE I

Results of fitting of the equation $\frac{d-d_{0}}{m}=A_{0}+A_{1} m^{\frac{1}{2}}+A_{2} m^{2}+A_{3} m^{\frac{3}{2}}$ (where $d$ and $d_{0}$ - the densities of a solution and water, respectively, $m-$ molality of the salt) for the system water + tetramethylammonium chloride. $R$ - correlation coefficient, $S D$ - standard deviation.

\begin{tabular}{c|c|c|c|c|c|c}
\hline \hline $\begin{array}{c}\text { Tempera- } \\
\text { ture }\left[{ }^{\circ} \mathrm{C}\right]\end{array}$ & $A_{0}$ & $A_{1}$ & $A_{2}$ & $A_{3}$ & $R$ & $S D$ \\
\hline 15 & 2.911409 & -1.043170 & 0.600422 & -0.103476 & 0.96495 & 0.03484 \\
20 & 2.500744 & -0.899087 & 0.578119 & -0.101498 & 0.97163 & 0.02468 \\
25 & 2.335628 & -1.052735 & 0.697306 & -0.121529 & 0.98451 & 0.02455 \\
30 & 2.217741 & -1.238805 & 0.831280 & -0.143795 & 0.99176 & 0.02370 \\
35 & 2.057403 & -1.250893 & 0.890300 & -0.159214 & 0.99798 & 0.01321
\end{tabular}

\subsection{Acoustic measurements}

The experimental sound velocities in the system under test are collected in Table II. From sound velocity (c) and density (d) data, using Laplace formula $\beta=\left(c^{2} d\right)^{-1}$ we calculated the adiabatic compressibility coefficients $(\beta)$ of solutions. They are in excellent agreement with the literature data [12]. Figure 1 shows the plots of the compressibility coefficient vs. concentration in the system under test for different temperatures.

The concentration corresponding to the intersection of compressibility isotherms changes with temperature (ranging from the solute mole fraction of $X \approx 0.035$ at $303 \mathrm{~K}$ to $X \approx 0.065$ at $288 \mathrm{~K}$ ). It should be noted that the intersection occurs in concentrations very close to those of the minima of compressibility, the fact never observed in typical water + organic solute systems studied before.

\subsection{Positron annihilation}

The experimental lifetime spectra were resolved into three components, each characterized by its lifetime $\left(\tau_{1}, \tau_{2}\right.$, and $\left.\tau_{3}\right)$ and intensity $\left(I_{1}, I_{2}\right.$, and $\left.I_{3}\right)$. Note that $I_{1}+I_{2}+I_{3}=100 \%$. The number of components was chosen arbitrarily, basing only on the quality of the fitting, while only the long-lived one $\left(\tau_{3}\right.$ and $\left.I_{3}\right)$, 
TABLE II

Experimental sound velocities in the solutions of tetramethylammonium chloride in water.

\begin{tabular}{l|c|c|c|c|c}
\hline $\begin{array}{l}\text { Mole fraction } \\
\text { of }\left(\mathrm{CH}_{3}\right)_{4} \mathrm{NCl}\end{array}$ & $15^{\circ}$ & $20^{\circ}$ & $25^{\circ}$ & $30^{\circ}$ & $35^{\circ}$ \\
\hline 0.0 & 1470.0 & 1483.0 & 1497.0 & 1510.0 & 1521.0 \\
0.001056 & 1471.1 & 1491.1 & 1507.0 & 1518.7 & 1526.3 \\
0.002080 & 1479.1 & 1496.3 & 1511.0 & 1523.2 & 1533.0 \\
0.004569 & 1496.4 & 1511.8 & 1525.0 & 1536.0 & 1544.7 \\
0.008056 & 1513.1 & 1528.6 & 1541.9 & 1552.9 & 1561.9 \\
0.01407 & 1550.8 & 1562.5 & 1573.1 & 1582.5 & 1590.7 \\
0.01834 & 1576.0 & 1588.6 & 1598.4 & 1605.3 & 1609.3 \\
0.02499 & 1605.0 & 1616.4 & 1623.1 & 1628.6 & 1633.3 \\
0.02894 & 1626.0 & 1634.4 & 1642.5 & 1648.9 & - \\
0.06162 & 1709.4 & 1711.8 & 1713.5 & 1714.5 & 1714.9 \\
0.07989 & 1715.5 & 1715.4 & 1714.9 & 1713.9 & 1712.5 \\
0.10389 & 1688.1 & 1685.6 & 1682.7 & 1679.4 & 1675.8 \\
0.12036 & 1643.3 & 1639.6 & 1635.6 & 1631.3 & 1626.7 \\
0.15690 & 1458.6 & 1453.0 & 1447.1 & 1441.0 & 1434.6
\end{tabular}

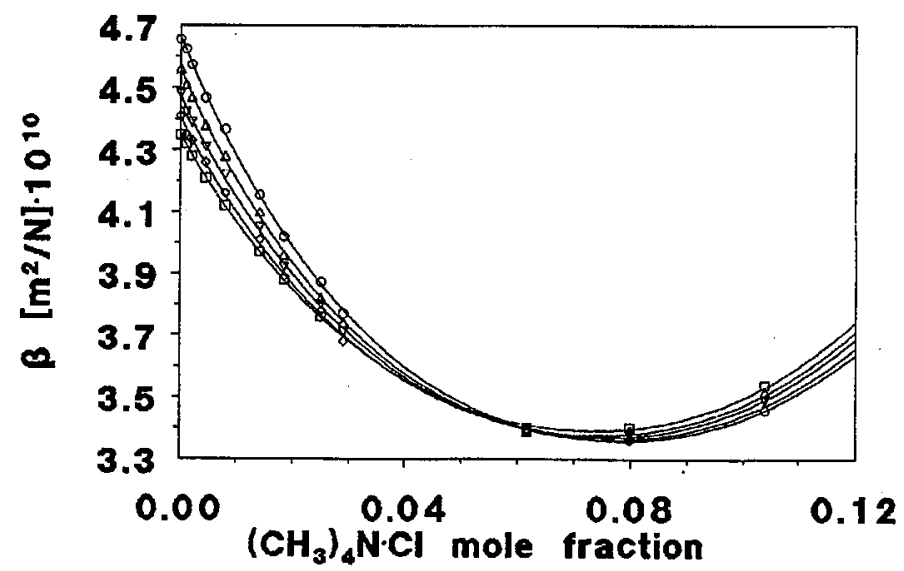

Fig. 1. Adiabatic compressibility coefficients vs. mole fraction of tetramethylammonium chloride in water: $\bigcirc-15^{\circ} \mathrm{C}, \triangle-20^{\circ} \mathrm{C}, \nabla-25^{\circ} \mathrm{C}, \diamond-30^{\circ} \mathrm{C}, \square-35^{\circ} \mathrm{C}$.

attributed to ortho-positronium annihilation, is of interest for further discussion. The short-lived components are a sum of different annihilation mechanisms and their more detailed analysis seems impossible. It should be strongly noted that our 
calculations yielded almost the same character of the concentration dependence of the long-lived parameter, but the short-lived one often lost a physical sense when we assumed more than two components. The results of annihilation experiments are collected in Table III.

\section{TABLE III}

Experimental parameters of positron annihilation spectra of the solutions of tetramethylammonium chloride in water. Only the long-lived component (subscript 3) can be useful for interpretation of the structure of liquid.

\begin{tabular}{l|c|c|c|c|c|c}
\hline \hline $\begin{array}{l}\text { Mole fraction } \\
\text { of }\left(\mathrm{CH}_{3}\right)_{4} \mathrm{NCl} \\
\text { in water }\end{array}$ & $\tau_{1}[\mathrm{~ns}]$ & $I_{1}[\%]$ & $\tau_{2}[\mathrm{~ns}]$ & $I_{2}[\%]$ & $\tau_{3}[\mathrm{~ns}]$ & $I_{3}[\%]$ \\
\hline 0 & 0.331 & 60.6 & 0.734 & 15.2 & 1.86 & 24.2 \\
0.001056 & 0.323 & 58.9 & 0.623 & 21.0 & 1.86 & 20.1 \\
0.002080 & 0.331 & 59.9 & 0.689 & 17.4 & 1.88 & 22.8 \\
0.004569 & 0.333 & 62.9 & 0.637 & 18.1 & 1.85 & 19.0 \\
0.008056 & 0.346 & 69.1 & 0.771 & 13.3 & 1.90 & 17.6 \\
0.01407 & 0.332 & 61.3 & 0.599 & 19.6 & 1.85 & 19.1 \\
0.01834 & 0.344 & 68.0 & 0.726 & 13.7 & 1.88 & 18.3 \\
0.02499 & 0.332 & 63.2 & 0.614 & 17.9 & 1.84 & 18.9 \\
0.02894 & 0.324 & 60.3 & 0.596 & 21.0 & 1.85 & 18.7 \\
0.06162 & 0.357 & 71.7 & 0.798 & 10.0 & 1.90 & 18.3 \\
0.07989 & 0.280 & 40.5 & 0.479 & 40.4 & 1.84 & 19.2 \\
0.1039 & 0.356 & 70.5 & 0.724 & 10.5 & 1.90 & 19.0 \\
0.1204 & 0.356 & 70.6 & 0.724 & 10.5 & 1.90 & 19.0 \\
0.1569 & 0.293 & 45.7 & 0.490 & 35.1 & 1.88 & 19.2 \\
0.2926 & 0.282 & 39.3 & 0.456 & 41.8 & 1.92 & 18.9
\end{tabular}

The obtained lifetimes of the long-lived component $\tau_{3}$, plotted vs. concentration of tetramethylammonium chloride are shown in Fig. 2, while the corresponding intensities $I_{3}$ are shown in Fig. 3. For comparison, our previous results obtained for water + ethanol mixtures [13] are included in these figures (broken lines).

The system water + ethanol was chosen as a typical hydrophobic one, where clathrate-like structures are undoubtedly formed in liquid phase. In the vicinity of the concentration corresponding to the stoichiometry of clathrate hydrates (ethanol mole fraction $X \approx 0.05$ ), the lifetime of the long-lived component $\tau_{3}$ passes through a plateau, while the intensity $I_{3}$ - through a flat maximum. None of these features are observed in the title system.

\section{Discussion}

According to the hypothesis of Endo [14], the intersection of the compressibility isotherms is determined by the stoichiometry of clathrate hydrates (if formed). 


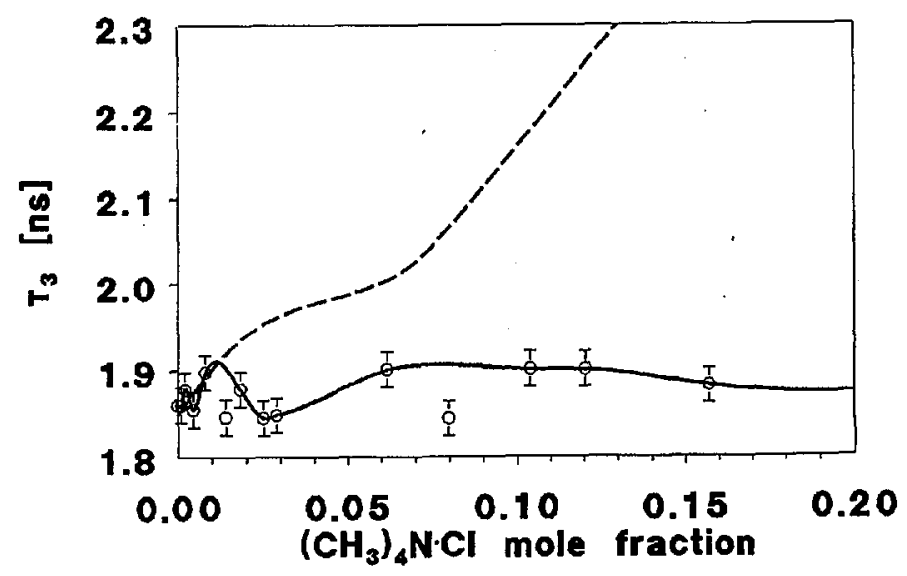

Fig. 2. Lifetimes of the long-lived component of annihilation spectra vs. tetramethylammonium chloride concentration in water (room temperature). Broken line: the results for aqueous solutions of ethanol [13].

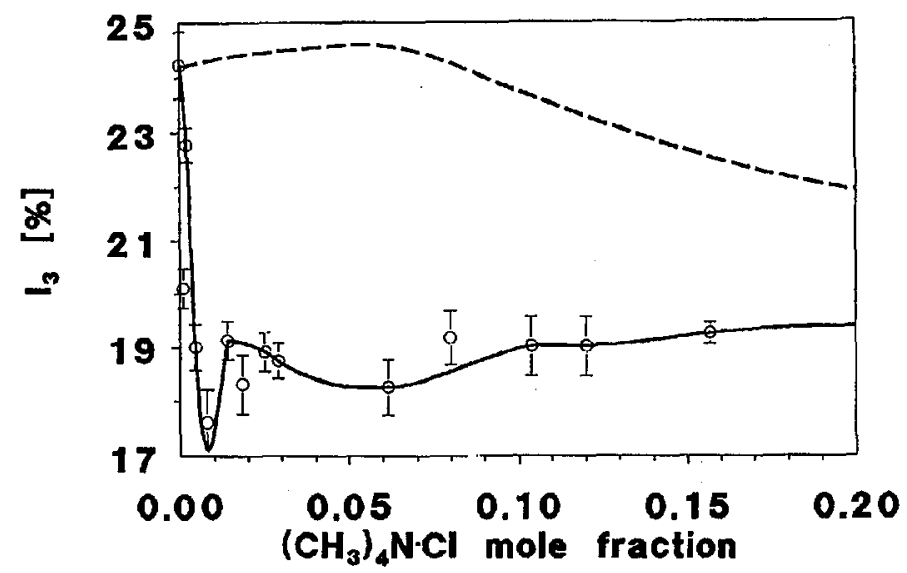

Fig. 3. Intensities of the long-lived component of annihilation spectra vs. tetramethylammonium concentration in water (room temperature). Broken line: the results for aqueous solutions of ethanol [13].

In the case of the title system, however, the Endo rule fails: the mole fraction corresponding to the intersection is $\approx 0.066$ at $288 \mathrm{~K}$, and $\approx 0.035$ at $303 \mathrm{~K}$, suggesting that the hydrate stoichiometry changes with temperature from $\mathrm{X} \cdot 14 \mathrm{H}_{2} \mathrm{O}$ at $288 \mathrm{~K}$ to X $\cdot 27 \mathrm{H}_{2} \mathrm{O}$ at $303 \mathrm{~K}$, while that of Dyadin [3] (obtained from phase diagrams) should be close to $\mathrm{Me}_{4} \mathrm{~N}^{+} \cdot(15-17) \mathrm{H}_{2} \mathrm{O}$ (assuming that the stoichiometry of tetramethylammonium chloride is the same as that of fluoride). Moreover, the 
fact that the composition corresponding to the intersection point is temperature dependent resembles the systems where hydrophilic hydration dominates: see for example the results for water + methanol [15], water + formamide [16] or water + ethylene glycol [17]. Thus, ultrasonic results give no evidence for formation of clathrate-like hydrates in liquid phase. The same conclusion can be drawn from positron annihilation results.

The above does not mean that Dyadin's findings concerning the stoichiometry of the clathrate-type hydrates of the tetramethylammonium cation are wrong. The intersection of the compressibility isotherms depends strongly on temperature. This fact could allow us to suppose that the stoichiometry is changing, i.e. formation of hydrates is not complete. For the system tested it seems reasonable to assume that the hydrates are very labile and are formed only in the presence of large excess of water.

It is worth noticing that the compressibility decreases with concentration, reaching at the intersection a value $\beta=3.4 \times 10^{-10} \mathrm{~m}^{2} \mathrm{~N}$, which is much lower than that of solid clathrates, determined by von Stackelberg [18] (for the type II clathrate hydrates approximately $4 \times 10^{-10} \mathrm{~m}^{2} / \mathrm{N}$ ). Moreover, the changes of annihilation parameters were observed, as far, only in classical clathrate-forming systems like water + alcohols [19]. The system under test contains, however, an ionic component. The above leads to conclusion that only partial hydration of cations occurs and/or ionic hydration influences strongly the annihilation parameters in solution.

The effect of chlorine anions, as well as their hydration, on the annihilation parameters remains unknown, too. Worse still, other processes are possible in aqueous solutions of tetraalkylammonium salts. They are, among others: (a) the effect of the charge of the tetramethylammonium cation on the water structure around it, (b) cation-cation interactions as observed by proton magnetic resonance in low solute concentrations [20], (c) formation of aggregates or ionic pairs, also those involving chlorine anions, etc.

Any comparison could be very difficult, too, even in a series of tetraalkylammonium chlorides. It was found from NMR investigations that tetramethylammonium cation is a structure-breaker, while tetrapropyl- and tetrabutylammonium ones are structure-makers [21]. In fact, our results are in contradiction with this classification.

In particular, tetraalkylammonium salts can form different hydrates, differing in their stoichiometry and structure. The above was confirmed by Turner et al. who found, from neutron diffraction for $2 \mathrm{M}$ tetramethylammonium chloride, that a range of water molecule orientations around the cation are possible [22]. Formation of different clathrate-like structures in aqueous solutions of tetrabutylammonium chloride was also proved by Jákli and Marko [23, 24]. From differential vapour pressure and molar volume measurements it was concluded that at low concentrations the stoichiometry of hydrates is close to $\mathrm{X} \cdot 63 \mathrm{H}_{2} \mathrm{O}$, while at higher concentrations it is transferred to $\mathrm{X} \cdot 33 \mathrm{H}_{2} \mathrm{O}$. Competition of different structures could also influence the observed character of annihilation parameters, as was found by us for tetrabutylammonium bromide + water [25]. 
Finally, the large size of the tetraalkylammonium cations makes formation of clathrate-like structures difficult. The stoichiometries given by Dyadin [3] are different than those known for solid clathrates. Note also that Kaatze et al. found

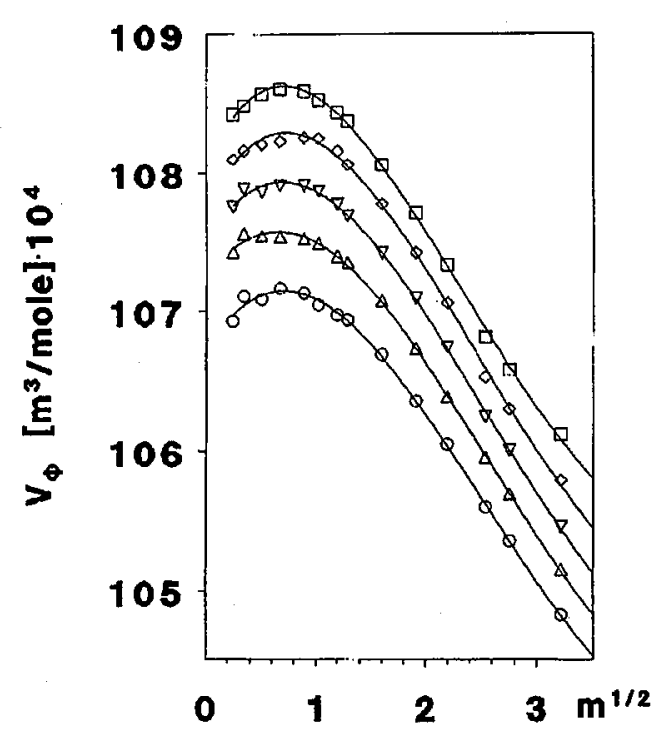

Fig. 4. Apparent molar volumes of the solute vs. square root of its molarity. For description refer to Fig. 1.

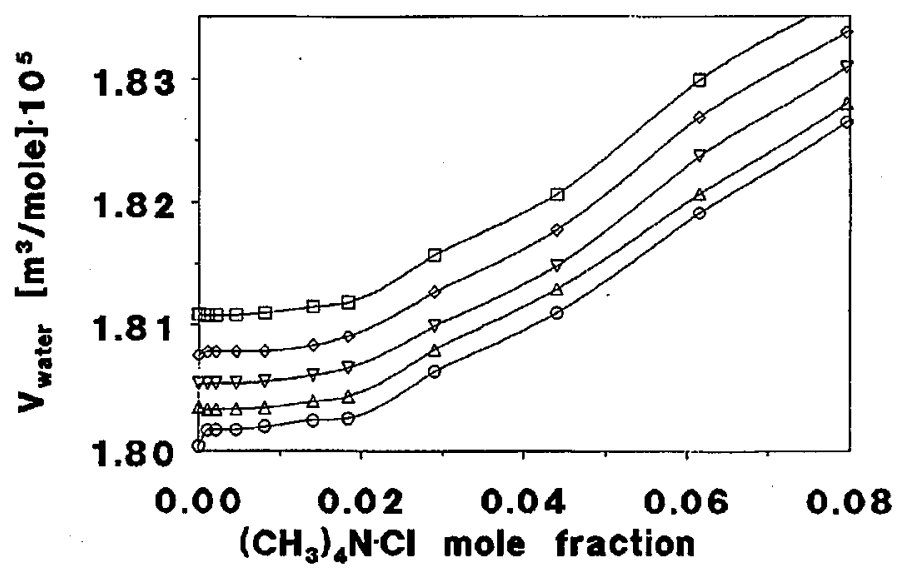

Fig. 5. Partial molar volumes of water in the solutions under tests. For description refer to Fig. 1. 
that hydrophobic interaction around $n$-alkylammonium ions is more pronounced than around tetraalkylammonium ones [26]. Finally, ultrasonic absorption results in aqueous tetraalkylammonium bromides give strong evidence that in aqueous solution the tetramethylammonium cation does not form clathrate-like hydrates at all [27] (while the tetrapropyl- and tetrabutylammonium ones do).

One could add to the difficulties described above the question about structural changes occurring in very low solute concentrations. Apparent molar volumes of an ionic solute should change linearly with square root of molarity, but in the system under consideration they pass through a flat maximum at, approximately, 0.01 mole fraction (Fig. 4), becoming linear above $X \approx 0.02$. It is worth noting that such maxima were not observed in aqueous solutions of tetrapropyl- and tetrabutylammonium salts; instead of this in these systems deep minima were found at $X \approx 0.02$ and more [28].

Even more interesting seems the partial molar volume of water in the system investigated, plotted vs. the salt concentration (Fig. 5). The plateau ending at $X \approx 0.02$ suggests initial enforcement of original solvent structure when adding the solute, i.e. structure-making behaviour.

Figures 4 and 5 suggest, surprisingly, that tetramethylammonium cation behaves as a structure-maker, but its structure-making effect ends at a very low concentration of $X \approx 0.02$.

Thus, the problem remains unsolved. On one hand, tetramethylammonium chloride behaves as a structure-breaker, with some little suggestions on formation of labile clathrate-like hydrates; on the other hand, it seems to be a structure-maker, weakly hydrated through electrostatic interactions and forming easily ionic pairs in higher concentrations.

\section{Conclusions}

1. The ultrasonic results (compressibility vs. concentration) suggest a non-typical stoichiometry of hydrates and/or rather hydrophilic character of hydration.

2. The concentration dependences of positron annihilation parameters (lifetimes and intensities of the long-lived component of annihilation spectrum) are substantially different than those determined for the known clathrate-forming systems.

3. The literature data suggest, however, that clathrate-like hydrates are formed in the title system.

4. It seems that ionic interactions influence the quantities observed by us, and/or that there are many different labile structures competing in the system.

\section{Acknowledgments}

A financial support from the Committee for Scientific Research (Poland) is acknowledged. 


\section{References}

[1] K. Jerie, A. Baranowski, B. Rozenfeld, J. Glinski, S. Ernst, Phys. Scr. 35, 729 (1987) and papers cited therein.

[2] Gy. Jákli, A. Baranowski, K. Jerie, J. Gliński, K. Orzechowski, Bull. Pol. Acad. Sci.: Chem. 42, 71 (1994).

[3] Yu.A. Dyadin, Supramolec. Chem. 6, 59 (1995).

[4] S.Sh. Byk, Yu.F. Makogon, V.I. Fomina, in: Gazovye Gidraty, Izd. Khimia, Moskva 1980, p. 12 and further (in Russian).

[5] W.-Y. Wen, J. Solution Chem. 2/3, 253 (1973).

[6] J.-Y. Huot, C. Jolicoeur, in: The Chemical Physics of Solvation. Part A. Theory of Solvation, Eds. R.R. Dogonadze, E. Kalman, A.A. Kornyshev, J. Ulstrup, Elsevier, New York 1985, p. 417.

[7] H.S. Frank, M.W. Evans, J. Chem. Phys. 13, 478 (1945).

[8] H.S. Frank, W.Y. Wen, Discuss. Faraday Soc. 24, 133 (1957).

[9] D.W. Davidson, in: Water. A Comprehensive Treatise, Ed. F. Franks, Vol. 2, Plenum Press, New York 1973, Ch. 3, p. 115.

[10] K. Jerie, A. Baranowski, B. Rozenfeld, S. Ernst, J. Gliński, Acta Phys. Pol. A 64, 77 (1983).

[11] S. Ernst, J. Gliński, B. Jeżowska-Trzebiatowska, Acta Phys. Pol. A 55, 501 (1979).

[12] B.E. Convay, R.E. Verrall, J. Phys. Chem. 70, 3952 (1966).

[13] K. Jerie, A. Baranowski, S. Ernst, J. Gliński, Acta Phys. Pol. A 69, 81 (1986).

[14] H. Endo, Bull. Chem. Soc. Japan 16, 1586 (1973).

[15] K. Jerie, A. Baranowski, B. Rozenfeld, S. Eırst, B. Jeżowska-Trzebiatowska, J. Gliński, Acta Phys. Pol. A 66, 167 (1984).

[16] K. Jerie, A. Baranowski, J. Gliński, K. Orzechowski, Acta Phys. Pol. A 85, 563 (1994).

[17] A. Baranowski, K. Jerie, J. Gliński, K. Orzechowski, Acta Phys. Pol. A 88, 59 (1995).

[18] M. von Stackelberg, H. Müller, Z. Elektrochem. 58, 25 (1954).

[19] K. Jerie, A. Baranowski, B. Rozenfeld, J. Gliński, S. Ernst, Phys. Scr. 35, 729 (1987).

[20] G. Kabisch, Ber. Bunsenges. Phys. Chem. 80, 602 (1976).

[21] M.-M. Marciacq-Rousselot, A. de Trobriand, M. Lucas, J. Phys. Chem. 76, 1455 (1972).

[22] J.Z. Turner, A.K. Soper, J.L. Finney, J. Chem. Phys. 102, 5438 (1995).

[23] Gy. Jákli, L. Marko, ACH-Models in Chemistry 132, 225 (1995).

[24] Gy. Jákli, L. Marko, Thermochim. Acta 259, 23 (1995).

[25] Gy. Jákli, A. Baranowski, K. Jerie, J. Gliński, K. Orzechowski, Nukleonika 42, 123 (1997).

[26] U. Kaatze, C.H. Limberg, R. Pottel, Ber. Bunsenges. Phys. Chem. 78, 555 (1976).

[27] M.J. Blandamer, M.J. Foster, N.J. Hidden, M.C.R. Symons, Trans. Faraday Soc. 64, 3247 (1968).

[28] W.-Y. Wen, S. Saito, J. Phys. Chem. 68, 2639 (1964). 